\title{
The design of focal plane splitting unit in a hyperspectral lidar system
}

\author{
Qian Liyong1 2, Wu Decheng ${ }^{1}$, Zhou Xiaojun'1, Zhong Liujun'1, Wei Wei', Deng Qian ${ }^{12}$, Chu \\ Yufei $^{12}$, Liu Dong ${ }^{1 *}$, Wang Yingjian ${ }^{12}$
}

1. Key Laboratory of Atmospheric Optics, Anhui Institute of Optics and Fine Mechanics, Chinese Academy of Sciences, Hefei, Anhui 230031

2.University of Science and Technology of China, Hefei, Anhui 230026

*Email:dliu@aiofm.ac.cn

\begin{abstract}
A hyperspectral lidar system with focal plane splitting unit is proposed, including an off-axis receiving telescope, a grating spectrometer, and a single-tube detector array. The spectrum of the system covers $380-930 \mathrm{~nm}$, and is separated by grating spectrometer. The microlens-fiber coupling system guides the echo signal of 50 channels into each detector. The system solves the data processing problem of the bandwidth and gain the line array and area array detector in traditional hyperspectral lidar. And it also meets the requirement of the high efficiency splitting coupling and weak signal acquisition and detection.
\end{abstract}

\section{INTRODUCTION}

Airborne/space-borne lidar usually works in a single-wavelength mode to acquire the earth surface data which contained single-wavelength echo intensity information ${ }^{[1,2]}$. In order to make better use of these observation capability in the existing lidar technology and scene classification, the intensity information and three-dimensional information of single-wavelength laser echo signals are combined to use ${ }^{[3]}$. Based on the backscattered signal reflected from the laser footprint, traditional method classifies the features such as road, grassland, crops, etc. The measurement based on the intensity information of the laser footprint to detect large rocks and cliffs in the forest area needs to get more accuracy of the mountain terrain data ${ }^{[4]}$. Seldom studies fundamentally improve the lack of physical detection capability of airborne lidar due to a single wavelength ${ }^{[5]}$. Although it has outstanding advantages in the acquisition of information in three-dimensional space, it is limited by the single-wavelength detection capability in terms of the physical property detection classification and state of the ground object. Compared with traditional passive remote sensing methods such as multi-hyperspectral imaging, it cannot reflect the essential advantages ${ }^{[6,7]}$.

According to the fast application of lidar and its technical characteristics, multi-spectral and multi-wavelength lidar remote sensing is carried out. This research with high spatial resolution detection capability will have important value and application prospects in the detect physical properties ${ }^{[8,9]}$. Just as passive optical remote sensing as a trend from a full-color camera to a multi-spectral camera-hyperspectral camera, the lidar geodetic observation technology is also shown a trend from single wavelength to double wavelength to multiple wavelengths.

This paper proposes a new type of Earth observation high-spectral lidar technology. The spectrum of the lidar system covers from 380 to $930 \mathrm{~nm}$ at wavelength. Due to the transmitted high-spectral laser, it can received the spectral and the collocated elevation information which improves the system's ability to distinguish vegetation, soil, rock and other ground objects which can be applied to forest survey, agricultural monitoring, land monitoring, resource exploration and other fields in a rapid, economical and accurate manner. 


\section{System Introduction}

As shown in Figure 1, by using the focal plane efficient spectroscopic technology based on microlens and fiber array, the wideband echo signals are efficiently divided into different channels detected by the high-speed, high-sensitivity detectors through optical fibers. Amplitude and dynamics of wide-spectrum laser echo signals adopt APD and PMT hybrid array detection technology to solve the problem of hyperspectral fast imaging under weak light conditions, realizing the full coverage detection of wide-spectrum bands of detection targets. Research on optical noise reduction technology should be used background light suppression technology to improve the detection performance of hyperspectral lidar. The design goal requires that the laser spectral band covers 380-930nm, and the hyperspectral lidar imaging detection with a band number of not less than 50 channels is realized.

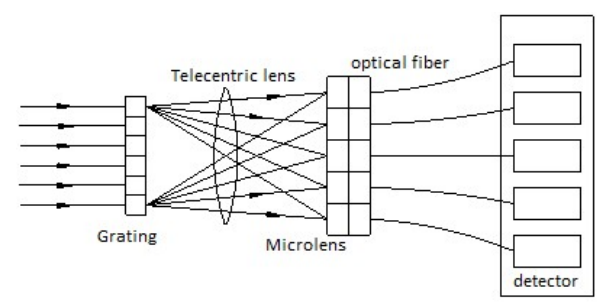

Figure 1 overall system design

\section{KEY TECHNOLOGY}

\subsection{Grating spectrometer}

As shown in Fig.2, the grating spectrometer is a spectroscopic device in the hyperspectral lidar, which mainly includes a small aperture diaphragm, a collimated off-axis parabolic mirror, a grating, and a telecentric spectral focusing lens. In order to improve the quality of remote sensing imaging, 380-930nm laser wide-spectrum coverage is used. The scattered echo light signal passing through the aperture pupil is collimated by a collimated off-axis parabolic mirror and then sent to the grating for splitting. And the telecentric lens put the spectrally separated spectrum refocuses.

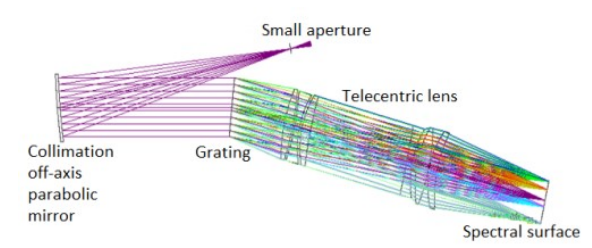

Figure 2 Optical design of grating spectrometer

The aperture is used to limit the receiving field of view in the receiving telescope, and the apertures are blackened on both sides to reduce the scattered light generated by the apertures of the apertures. An off-axis parabolic mirror is used to collimate the full-band echo optical signal through the aperture stop. Compared with the transmissive collimation method, the off-axis parabolic mirror can well collimate the full-band echo signal, which can reduce the dispersion of the focal plane of the grating focal plane because of the insufficient collimation parallelism. Thereby the spectral resolution could be improved.

Under the premise of satisfying the spectral resolution, the system is made more compact as much as possible, and the transmission grating is selected as the spectroscopic device. The focusing mirror behind the grating adopts a transmission focusing mirror system, which can effectively reduce the focusing spot aberration of each spectral channel after grating splitting to improve the spectral resolution ${ }^{[10]}$. In addition, the focusing mirror system adopts the structural form of the image telecentric system, ensuring the main rays of each spectral channel in perpendicular to the surface of the microlens-fiber detection array, effectively matching the receiving numerical aperture and improving the optical coupling efficiency.

According to the existing parameters, the spectral range is from 380 to $930 \mathrm{~nm}$ at wavelength, and the grating transmitted light is centered on the wavelength of $632 \mathrm{~nm}$. The diffraction effect of the grating is the effect of single-slit diffraction and multi-slit interference superposition. For single-slot interference, phase 
there is an optical path difference $\Delta L=2 d \sin \theta$ between the adjacent groove faces in the transmission direction, Satisfy $2 d \sin \theta=\lambda$, $\lambda$ is a class 1 blaze wavelength, and the grating equation is:

$$
\sin \alpha+\sin \beta=m \lambda / d
$$

Where $\alpha$ and $\beta$ represent the angle of incidence and diffraction angle of the grating, respectively; $m$ is the diffraction order; $\lambda$ is the wavelength; $d$ is the grating constant. The relationship between the incident light wavelength $\lambda$ and the grating blaze angle $\alpha$ of the transmission grating is:

$$
\sin \alpha=\frac{m \lambda}{2 d}
$$

Therefore, the incident angle $\theta$ of the grating spectrometer is:

$\theta=\arcsin \left(\frac{m \lambda}{2 d}\right)=\arcsin \left(\frac{632 \times 10^{-6} \times 360}{2}\right)=6$.

Substituting the grating spectrometer line dispersion is:

$$
\frac{1}{D_{l}}=\frac{\cos \theta}{\text { line } \cdot f_{c}} \cdot 10^{6}=10 \mathrm{~nm} / \mathrm{mm}
$$

\subsection{Focus splitting system}

As shown in Fig. 3, each fiber corresponds to each microlens, and the grating splits the broad spectral signal with the spectral segment $N \sim M$ into $n+1$ spectral segments such as $N \sim N_{1} 、 N_{1} \sim N_{2} 、 \ldots N_{\mathrm{n}} \sim M$. Spectral resolution is $\frac{M-N}{n+1}$. In this paper, the spectrum covers from 380 to $930 \mathrm{~nm}$ at wavelength, achieving 50 channels with a spectral resolution of $10 \mathrm{~nm}$.

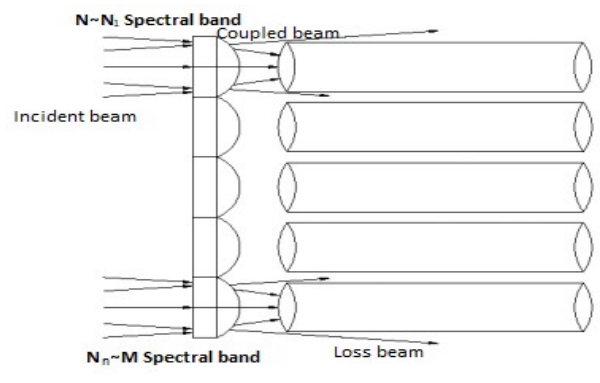

Figure 3 Microlens-fiber coupling system

The optical design of the microlens array is shown in Figure 4. It can be seen from the figure that after the microlens, the spot which concentrated on the end face of the fiber is equivalent to increasing the filling rate of the fiber array. Figure 5 shows the simulation results of the effect of the microlens on the spot energy distribution on the fiber's end face. It can be seen from Fig. 5 (a) and (b) that the energy of the spot is uniformly distributed without the addition of the microlens, and only about $50 \%$ of the energy is included in the effective clear aperture of the fiber, coupling the efficiency is $50 \%$. After adding the microlens, it can be seen from (c) and (d) that the energy of the spot is mainly concentrated in the center of the fiber, and contains about $90 \%$ of the spot energy in the range of about 100um in the center of the fiber. Therefore, after using the microlens array, the coupling efficiency of the fiber array can be increased from $50 \%$ to more than about $95 \%$.

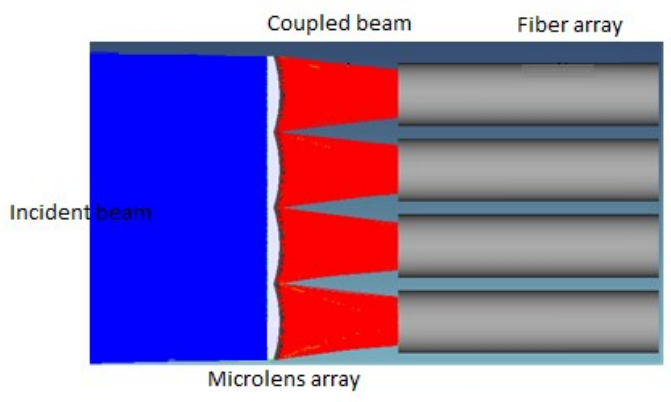

Figure 4 Microlens-fiber coupling design 

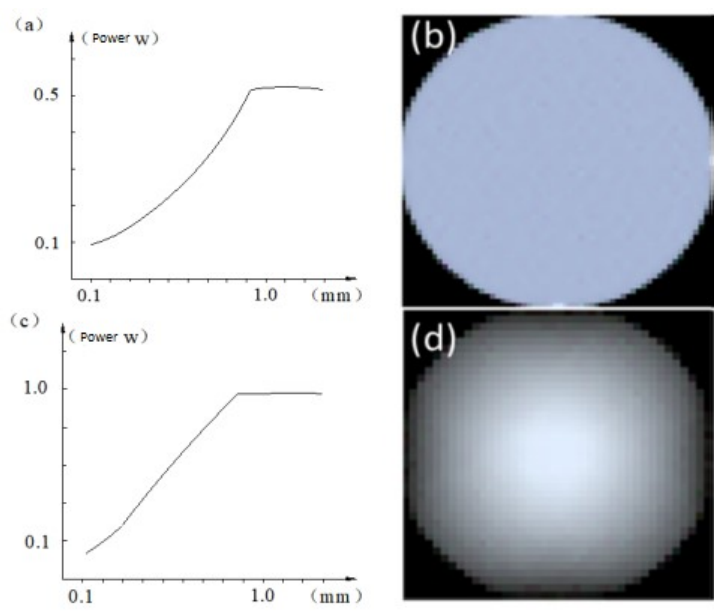

Figure 5 Spot energy distribution

\section{CONCLUSION}

In this paper, a new design of Spectroscopic system in hyperspectral lidar is proposed. The spectral coverage is $380-930 \mathrm{~nm}$, the spectral resolution is better than $10 \mathrm{~nm}$, and 50 channels of high-speed synchronous data acquisition are realized. Further work needs to complete the development of the system prototype, and realize the airborne flight test. This work could also obtain the hyperspectral laser detection data and simultaneously realize the cross-domain of the high-spectrum lidar successfully developed by demonstration prototype theory. It would truly promote the hyperspectral lidar to practical development.

\section{ACKNOWLEDGEMENTS}

Supported by National Key R\&D Program of China(2018YFB0504500).

\section{REFERENCES}

1. Song.S,et al, Isprs Journal of Photogrammetry \& Remote Sensing, 66(5): p. 672-682 (2011)

2. Elaksher, A.F, Optics and Lasers in Engineering, 46(7): p. 493-498 (2008)

3. Dalponte, M,et al, IEEE Transactions on Geoscience \& Remote Sensing, 46(5): p. 1416-1427 (2008)

4. Hakala, T, et al, Optics Express, 20(7): p. 7119-7127 (2012)

5. Nichol, C. J, et al, in Agu Fall Meeting. 2009.

6. Harding, D. J, et al, Remote Sensing of Environment, 76(3): p. 283-297 (2001)

7. Lovell, J.L, et al, Canadian Journal of Remote Sensing, 29(5): p. 16 (2003)

8. Bourliaguet, B, et al, GIS Applications, and Geology IX. 2009.

9. Lin, D, et al, International Journal of Applied Earth Observation \& Geoinformation,44: p. 136-143 (2016)

10.Helmut, R, et al, Optics Express, 17(6): p. 4268 (2009) 